\title{
Inhibition of ZEB1 leads to inversion of metastatic characteristics and restoration of paclitaxel sensitivity of chronic chemoresistant ovarian carcinoma cells
}

\author{
Jun Sakata ${ }^{1}$, Fumi Utsumi ${ }^{1}$, Shiro Suzuki ${ }^{1}$, Kaoru Niimi ${ }^{1}$, Eiko Yamamoto' ${ }^{2}$, Kiyosumi \\ Shibata $^{3}$, Takeshi Senga ${ }^{4}$, Fumitaka Kikkawa ${ }^{1}$ and Hiroaki Kajiyama ${ }^{1}$ \\ ${ }^{1}$ Department of Obstetrics and Gynecology, Graduate School of Medicine, Nagoya University, Nagoya, Japan \\ ${ }^{2}$ Department of Healthcare Administration, Graduate School of Medicine, Nagoya University, Nagoya, Japan \\ ${ }^{3}$ Department of Obstetrics and Gynecology, Banbuntane Hotokukai, Fujita Health University, Fujita, Japan \\ ${ }^{4}$ Division of Tumor Biology, Graduate School of Medicine, Nagoya University, Nagoya, Japan \\ Correspondence to: Hiroaki Kajiyama, email: kajiyama@med.nagoya-u.ac.jp \\ Keywords: epithelial ovarian carcinoma, epithelial-mesenchymal transition, ZEB1, chemoresistance, metastasis
}

Received: June 07, 2017

Accepted: July 25, 2017

Published: August 10, 2017

Copyright: Sakata et al. This is an open-access article distributed under the terms of the Creative Commons Attribution License 3.0 (CC BY 3.0), which permits unrestricted use, distribution, and reproduction in any medium, provided the original author and source are credited.

\section{ABSTRACT}

ZEB1, a member of the zinc-finger E-box binding homeobox family, is considered to play a crucial role in cancer progression and metastasis. In the current study, we investigated the role of ZEB1 in metastasis and chronic chemoresistance of epithelial ovarian carcinoma (EOC) cells. Using several EOC and acquired paclitaxel (PTX)resistant EOC cell lines, we investigated whether silencing ZEB1 led to a reversal of the chemoresistance and metastatic potential in vitro and in vivo. Subsequently, the expression of ZEB1 in EOC tissues and its association with the oncologic outcome were investigated. According to the immunohistochemical staining of EOC tissues, as the positivity of ZEB1 expression was increased, the overall survival of EOC patients became poorer ( $P=\mathbf{0 . 0 0 2 2}$ for trend). Additionally, cell migration and invasion were significantly decreased by ZEB1 silencing in both PTX-sensitive and PTX- resistant cells. Although PTX-sensitivity was not changed by silencing ZEB1 in parental EOC cells, the depletion of ZEB1 made the PTX-resistant EOC cells more sensitive to PTX treatment. In an animal model, mice injected with ZEB1-silencing PTX-resistant cells survived for longer than the control cell-injected mice. Although the intravenous injection of PTX did not affect the tumor weight of shCtrl cells, the tumor weight of shZEB1 cells was significantly reduced by PTX treatment. The current data indicate the possible involvement of ZEB1 in the metastasis and paclitaxel resistance of EOC, and suggest that targeting this molecule may reverse the malignant potential and improve the oncologic outcome for EOC patients.

\section{INTRODUCTION}

Epithelial ovarian carcinoma (EOC) is a major cause of mortality among all gynecological malignancies [1]. The recent Cancer Statistics estimated that 238,700 women were newly diagnosed with EOC, and 151,900 died of this tumor worldwide [2]. The prognosis of patients with EOC is most likely related to the degree of peritoneal dissemination [3-5]. Since EOC often remains silent in clinical situations, the majority of patients show aggressive peritoneal dissemination at the time of diagnosis [6]. Reflecting the cell biology of EOC, despite the fact that complete clinical remission can be achieved in approximately $80 \%$ of these patients owing to cytoreductive surgery, followed by systematic front-line chemotherapy, the majority of those clinical complete responders develop recurrent disease [7]. If all EOC tumor cells have sufficient sensitivity to conventional chemotherapy, they will disappear in due course. Nevertheless, since the sensitivity of tumors 
is actually heterogeneous, recurrence will eventually develop due to the remaining resistant tumor cells. EOC cells that have acquired chemoresistance may have hallmarks that favor easily spreading into the peritoneal cavity, resulting in an increased chance of adhering to the mesothelium and the enhanced formation of microscopic or macroscopic peritoneal metastasis in vivo [8]. Therefore, the clinical outcome of relapsed patients remains poor.

ZEB1, a member of the zinc-finger E-box binding homeobox ( $\mathrm{ZFH}$ ) family, is considered to play a crucial role in cancer progression and metastasis, it shows high-level expression in epithelial cancers, including prostate, hepatocellular carcinoma, lung, and pancreatic cancers, and its expression is correlated with a poor prognosis [9-11]. Through driving epithelial-mesenchymal transition (EMT), ZEB1 contributes to the metastasis of carcinoma cells, and prior studies demonstrated that ZEB1 conferred stemness and resistance [12]. Inhibition of ZEB1 reversed EMT and chemoresistance in chemoresistant human lung cancer cells [13]. In addition, interference with the ZEB1 function by the class I HDAC inhibitor mocetinostat led to the restoration of miR-203 expression, repressing stemness properties, and inducing sensitivity to chemotherapy [14]. Kikuchi et al demonstrated that Phenylbutyrate, a histone deacetylase antagonist that also exhibits antitumor activity sensitivity, was reported to be influenced by epigenetic expression alteration of ZEB1 in breast cancer cells [15]. This study proved that epigenetic regulation of ZEB1 may be a key biomarker for predicting resistance to breast cancer therapies. Furthermore, downregulation of ZEB1 by salinomycin increased the sensitivity of Mantle cell lymphoma cells to the cytotoxic effects of doxorubicin, cytarabine, and gemcitabine [16].

We previously demonstrated that chronic chemoresistance to paclitaxel (PTX) induced EMT and enhanced the peritoneal metastatic potential of EOC cells using a murine model [8]. Here, we aimed to clarify the role of ZEB1 in chemoresistance / metastasis, and clinical impact of ZEB1 expression in EOC by exploring: (i) ZEB1 expressions in various EOC cells and functions, including cell migration, invasion, and attachment to mesothelial cells, ii) ZEB1 expressions in two independent chronic PTX-resistant human EOC cell lines, which displayed a typical EMT phenotype, (iii) whether interfering ZEB1 expression restored sensitivity to PTX and exerted an anti-metastatic / chemoresistant potential, (iv) significance of ZEB1 expression in the peritoneal microenviroment displaying cell-to-cell communication between mesothelial and EOC cells, and (v) survival impact of ZEB1 expression in actual clinical samples. The possible function of the transcriptional factor as a facilitator of EOC metastasis is reported.

\section{RESULTS}

\section{Expression of ZEB1 correlated with unfavorable outcome of patients with EOC}

The ZEB1 immunoreactivity was classified into the four scoring types as described in "Materials and Methods" (Negative, weakly, moderately, and strongly positive expressions). Representative images of each histological feature are shown in Figure 1A-1H.

In several cases, the immunoexpressions of ZEB1 were identified in the stroma as well as carcinoma tissues. Of the 40 carcinomas, negative, weakly, moderately, and strongly positive ZEB1 immunoexpressions were observed in $7(17.5 \%), 14(35.0 \%), 11(27.5 \%)$, and 8 $(20.0 \%)$ patients, respectively. Compared with negative expression, positive ZEB1 expression predicted a significantly poorer overall survival \{Negative vs. weak, moderate, and strong $(P=0.0071)$ : Figure $1 \mathrm{I}\}$. Furthermore, as the positivity of ZEB1 expression was increased, the overall survival of EOC patients became poorer $(P=0.0022$ for trend: Figure $1 \mathrm{~J})$.

\section{ZEB1 involved in migration, invasion, and adhesion to mesothelial cells, and PTX sensitivity of EOC cells}

To investigate the role of ZEB1 in the malignant characteristics of EOC, we examined the expressions of ZEB1 in various EOC cells. ZEB1 was highly expressed in ES-2, TOV21G, A2780, and HEY cells. Moderate expression of ZEB1 was observed in SKOV3 and OV90 cells. We used ES-2 and SKOV3 cells, which showed high and moderate expression of ZEB1, respectively (Figure 2A). For further analyses, since ZEB1 is one of the major transcription factors that promote EMT in various cancer cells, we depleted ZEB1 in ES-2 and SKOV3 cells and examined the expression of E-cadherin as an epithelial marker, and vimentin and $\mathrm{N}$-cadherin as mesenchymal markers. ZEB1 knockdown did not affect the expression of marker proteins in ES-2 cells; however, in SKOV3 cells, an increase of E-cadherin and decrease of vimentin and $\mathrm{N}$-cadherin were clearly observed on ZEB1 depletion (Figure 2B). Similar tendencies were observed in TOV21G, OV90, and A2780 cells (Supplementary Figure 1). These results are consistent with the previous finding that ZEB1 is associated with EMT.

EMT is often associated with increases in the migratory ability and invasive potential of cancer cells. To examine the migration of EOC cells, we used transwell chambers whose lower surface of the filter was coated with fibronectin. As shown in Figure 2C and Supplementary Figure 2A, the migration of both ES-2 and SKOV3 cells was significantly reduced by ZEB1 knockdown. To determine cell invasion, we used Matrigel-coated transwell 
chambers. ZEB1 depletion also suppressed the invasive potential of both cell lines (Figure 2D and Supplementary Figure 2B).

The peritoneal mesothelium is a monolayer of epithelial cells covering the peritoneal cavity and forming serosal membranes. The attachment of EOC cells to mesothelial cells is a critical step for cancer dissemination over the mesothelium. We tested if ZEB1 was required for the promotion of EOC cell attachment to HPMCs. ES-2 or SKOV3 cells transfected with siRNAs were seeded onto confluent monolayers of HPMCs and the attached cells were evaluated. As shown in Figure 2E, 2F, the suppression of ZEB1 reduced the attachment of both EOC lines to HPMCs. These results show that ZEB1 is required for cell migration, invasion, and attachment to mesothelial cells.

We subsequently examined whether ZEB1 was associated with the acquisition of PTX-resistance in vitro. ES-2 and SKOV3 cells transfected with siRNAs were treated with different concentrations of PTX, and the surviving cells were evaluated by the MTT assay, as described in "Materials and Methods". In both lines, ZEB1 knockdown clearly increased the sensitivities to paclitaxel. The IC50 values of PTX were significantly reduced by ZEB1 depletion in both lines (Figure 2G, 2H).

\section{ZEB1 expression and its function in chronic PTX-resistant EOC cells}

To further confirm that ZEB1 was required for chemoresistance, we generated two independent PTXresistant cell lines using parental NOS2 and NOS3 cells, which showed low expression of ZEB1. We treated both cell lines for months with different concentrations of PTX and finally obtained highly PTX-resistant cells, NOS2TR and NOS3TR, respectively (Figure 3A). The morphology of NOS2TR and NOS3TR cells became spindle-
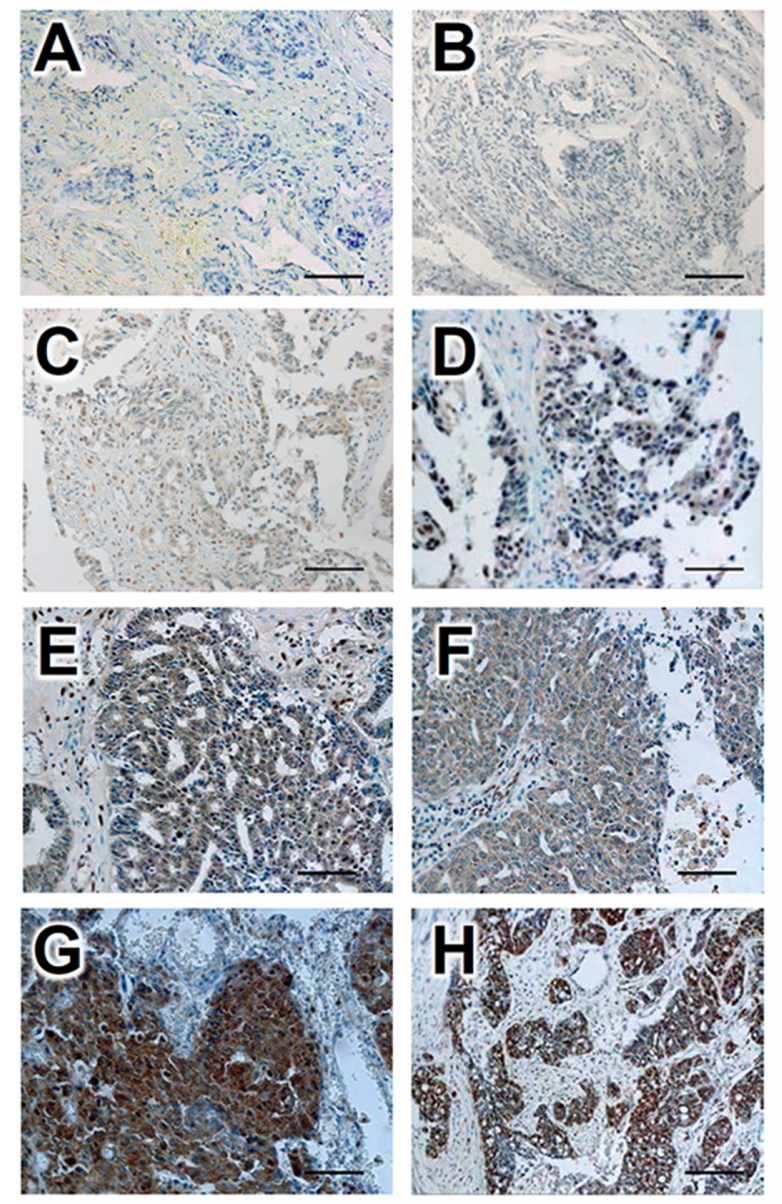
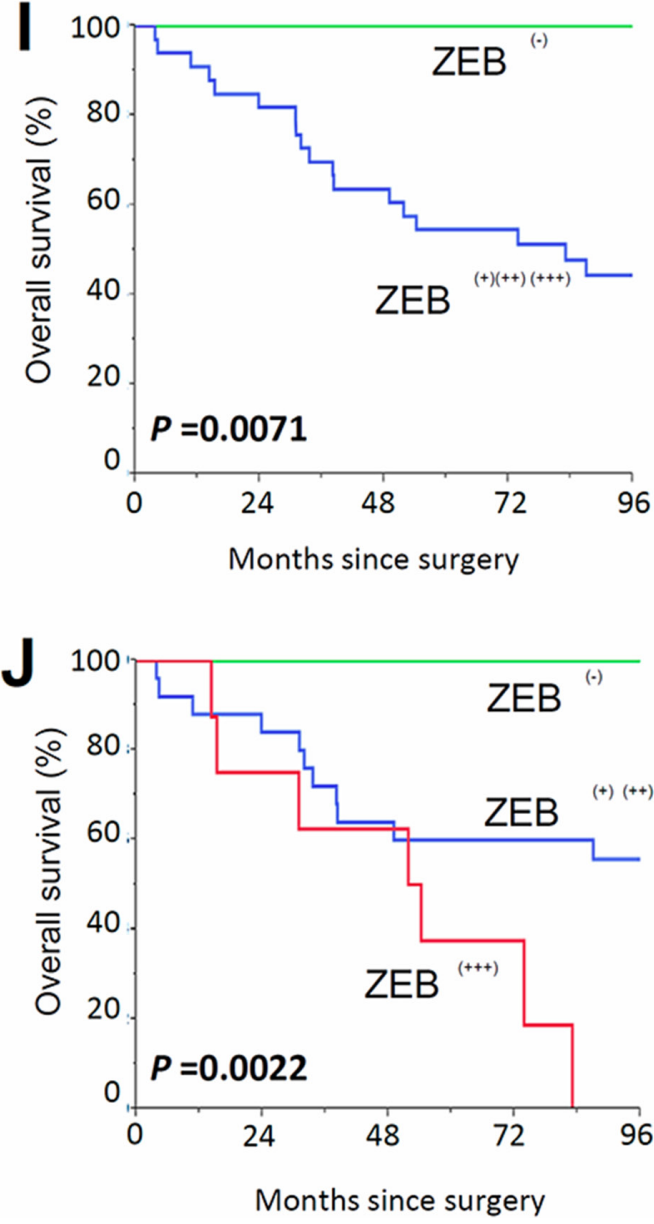

Figure 1: Survival impact of ZEB1 expression in EOC tissues. Immunoreactivity of ZEB1 observed in surgical EOC samples (paraffin sections), positive or negative expression of ZEB1 in EOCs. (A, B) negative, $(\mathbf{C}, \mathbf{D})$ weakly positive, (E, F) moderately positive, $(\mathbf{G}, \mathbf{H})$ strongly positive; magnification $\times 100$. (I, J) Kaplan-Meier overall survival curves for primary EOCs according to immunoexpression of ZEB1. Two-group comparison (I): Green line represents negative ZEB1 expression (negative: $N=7$ ). Blue line represents positive ZEB1 immunoexpression (weekly-strongly positive: $N=33)(P=0.0071)$. Three-group comparison (J): Green line represents negative ZEB1 expression (negative: $N=7$ ). Blue line represents positive ZEB1 immunoexpression (weekly-moderately positive: $N=28$ ). Red line represents positive ZEB1 immunoexpression (strongly positive: $N=8)(P=0.0022)$. 
shaped with disrupted cell-cell adhesion (Figure 3B). Nearly $80-100 \%$ of NOS2 and NOS3 cells died in the presence of $10 \mathrm{ng} / \mathrm{mL}$ of PTX, whereas approximately $40 \%$ of both lines were alive with $100 \mathrm{ng} / \mathrm{mL}$ of PTX (Figure 3C). NOS2TR and NOS3TR showed increased cell migration and invasion, compared with each parental cells line (Figure 3D). In addition, we examined whether chronic PTX-resistant cells showed the mesenchymal characteristics by immunoblot. As shown in Figure 3E, the expression of E-cadherin was decreased and a clear increase of vimentin was observed. Consistent with the enhanced migratory potential, ZEB1 expression was higher in NOS3TR cells than parental NOS3 cells. Furthermore, we examined the effect of silencing ZEB1 on both migration and invasion of chronic PTX-resistant EOC cells. As shown in Figure 3F, ZEB1 knockdown induced the enhanced expression of E-cadherin and downregulation of vimentin. Accordingly, migration and
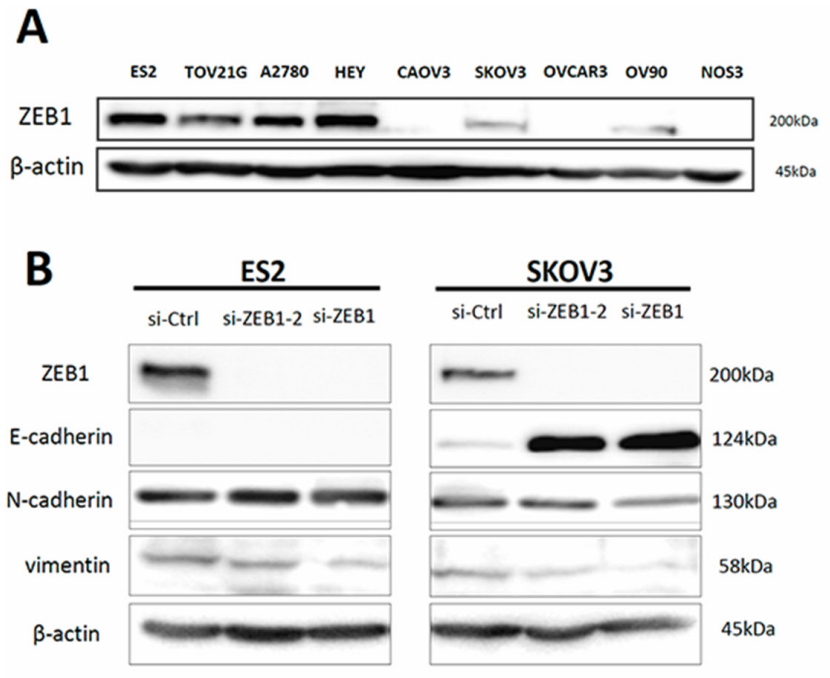

G

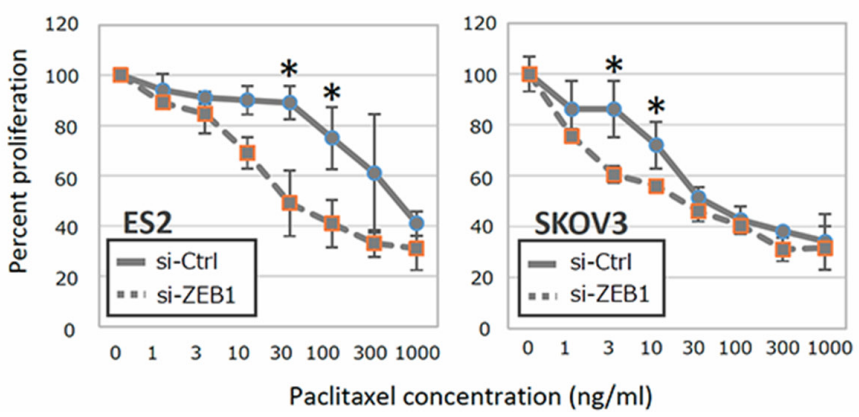

invasion were significantly decreased by ZEB1 silencing in NOS3TR cells (Figure 3G). A representative image of decreased cell migration is presented in Figure $3 \mathrm{H}$.

We also examined whether ZEB1 was required for the promotion of chronic PTX-resistant cell attachment to HPMCs. NOS3 and NOS3TR cells transfected with siRNAs were seeded onto the confluent monolayer of HPMCs and attached cells were evaluated. As shown in Supplementary Figure 3, suppression of ZEB1 significantly reduced the attachment of NOS3TR cells to HPMCs, although it did not affect NOS3 cells.

\section{Restoration of PTX-sensitivity by silencing ZEB1 expression in chronic PTX-resistant cells}

Next, we investigated whether the acquired PTX-resistance of NOS3TR was dependent on the increased expression of ZEB1. In parental NOS3 cells,
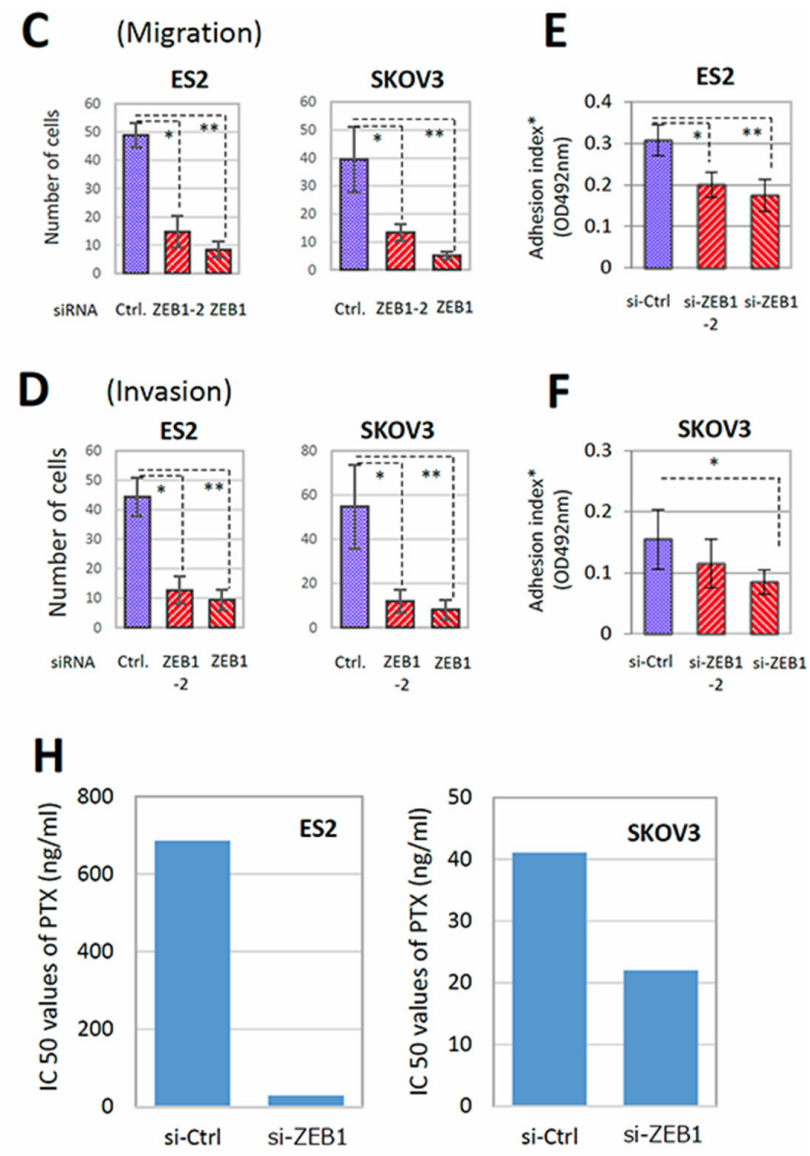

Figure 2: ZEB1 was involved in the migration, invasion, adhesion to mesothelial cells, and PTX sensitivity of EOC cells. (A) The expression of ZEB1 in various EOC cells. ZEB1 was highly expressed in ES-2, TOV21G, A2780, and HEY cells. (B) Depletion of ZEB1 in ES-2 and SKOV3 cells and influence on expression of E-cadherin as an epithelial marker, and vimentin and N-cadherin as mesenchymal markers. (C, D) Effect of ZEB1 silencing on the migratory ability (C) and invasive potential (D) of ES-2 and SKOV3 cells. Asterisks show significance $(P<0.05)$. (E, F) Effect of ZEB1 silencing on the attachment of EOC cells to the confluent monolayer of human peritoneal mesothelial cells (HPMCs) (E) ES2 cells, (F) SKOV3 cells). Asterisks show significance $(P<0.05)$. (G, H) Restoration of PTX-resistance in ES-2 and SKOV3 cells by ZEB1 silencing. The PTX-sensitivity assay using evaluated by the MTT assay (G). Asterisks show significance $(P<0.05)$. ZEB1 knockdown clearly increased the sensitivities to PTX. IC50 values of PTX were significantly reduced by ZEB1 depletion in ES-2 and SKOV3 cells $(\mathrm{H})$. 
the PTX-sensitivity was not changed by silencing of ZEB1 (Figure 4A). In contrast, the depletion of ZEB1 made NOS3TR cells more sensitive to PTX treatment (Figure 4B). The IC50 value of control siRNA-transfected NOS3TR cells was $41.8 \mathrm{ng} / \mathrm{mL}$, whereas that of ZEB1depleted NOS3TR was $6.2 \mathrm{ng} / \mathrm{mL}$. To further clarify the mechanism of PTX-sensitivity by ZEB1 knockdown, we examined the phosphorylation of ERK1 and Akt. As shown in Figure 4C, the phosphorylation of ERK1 and Akt was significantly decreased in ZEB1-depleted NOS3TR cells compared with that of control siRNA-transfected NOS3TR cells. However, this reduction was also observed in parental NOS3 cells. In addition, we examined the expression of cleaved PARP, which is an indicator of the induction of apoptosis. As shown in Figure 4D, the induction of cleaved PARP was more markedly observed in ZEB1-depleted NOS3TR cells compared with NOS3 cells. Furthermore, in the presence of PTX, the expression of cleaved PARP was significantly higher in ZEB1depleted NOS3TR cells compared with that of control siRNA-transfected NOS3TR cells (Figure 4E).

\section{Enhancement of ZEB1 expression by the microenvironmental production of TGF- $\beta$}

As previously mentioned, HPMCs serve as a protective barrier, being the front-line cellular barrier against disseminated EOC cells [17]. HPMCs typically shows an epithelial morphology with a cobblestone appearance (Figure 5A). TGF- $\beta$ has been identified as a representative inducer of EMT. We initially examined the
TGF- $\beta$ concentration in conditioned media of HPMCs, NOS3, NOS3TR, and HPMCs plus NOS3TR cells (co-culture condition). As shown in Figure 5B, TGF- $\beta$ production in NOS3TR cells was significantly higher than in parental NOS3 cells. However, it was synergistically higher under the co-culture condition with HPMCs. We subsequently tested the effect of TGF- $\beta$ in EMT-related characteristics in PTX-resistant NOS3TR cells. As a result, the addition of TGF- $\beta$ to NOS3TR cells led to a significantly enhanced migratory potential compared with parental NOS3 cells (Figure 5C). In addition, the presence of TGF- $\beta$ led to a more mesenchymal cell shape with the loss of cell polarity and decreased E-cadherin expressions, particularly in NOS3TR cells (Figure 5D). Furthermore, we subsequently examined whether the EMT, or TGF- $\beta$ signal-related proteins were altered in NOS3 and NOS3TR cells with or without TGF- $\beta$. The addition of TGF- $\beta$ resulted in increased ZEB1 expression with the enhanced expression of phosphorylated Smad2, particularly in NOS3TR cells (Figure 5E). Those results suggest that TGF- $\beta$ produced in the microenvironmental cell-to-cell communication contributes to the upregulation of ZEB1, thought to play a role in chronic PTX-resistance/ peritoneal metastasis.

\section{ZEB1 required for chemo-resistance to $\mathrm{PTX}$ in vivo}

To further extend our analysis, we performed in vivo analyses using nude mice. We generated NOS3TR cells that constitutively expressed control shRNA ( $\mathrm{shCtrl)}$ or ZEB1 shRNA (shZEB1) by retrovirus infection. Clear
A

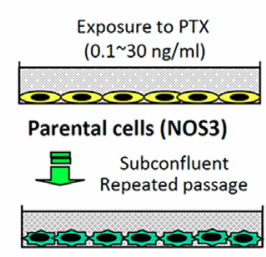

C Resistant cells (NOS3TR)

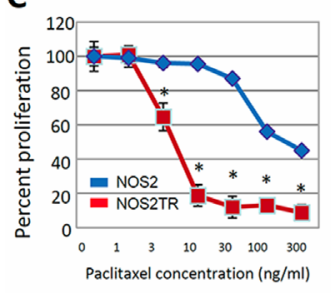

B
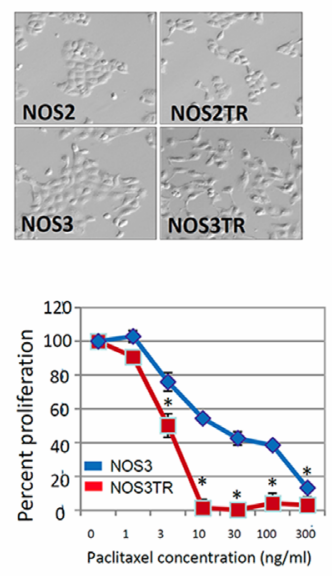
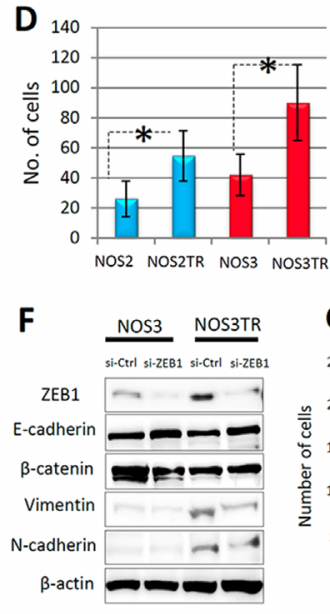

E

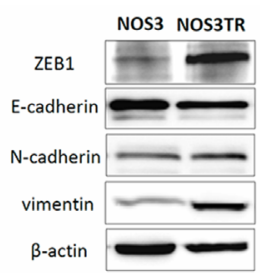

G

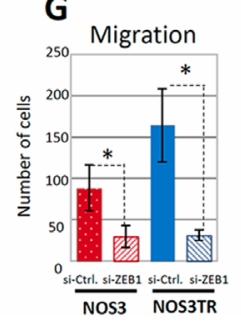

H

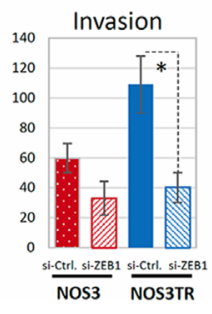

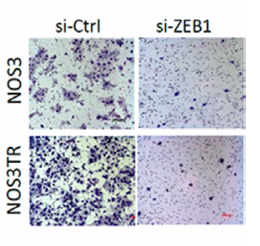

Figure 3: ZEB1 expression and function in chronic PTX-resistant EOC cells. (A) Generation of two independent PTX-resistant cell lines using NOS2 and NOS3 cells by continuous exposure to stepwisely increasing concentrations of PTX. (B) The morphology of two independent PTX-resistant cell lines (NOS2TR and NOS3TR cells: spindle-shaped). (C) PTX-sensitivity assay of the chronic PTX-resistant cells using evaluated by the MTT assay. ZEB1 knockdown clearly increased sensitivities to PTX. Asterisks show significance $(P<0.05)$. (D) The migratory potentials of NOS2TR and NOS3TR cells. Asterisks show significance $(P<0.05)$. (E) Western blot analysis showing the increased expression of ZEB1 and induced mesenchymal characteristics in acquired PTX-resistant cells (NOS3TR). (F) Western blot analysis: Induction of enhanced expression of E-cadherin and downregulation of vimentin by ZEB1 knockdown. (G) The effect of ZEB1 silencing on cell migration and invasion of NOS3 and NOS3TR cells. Asterisks show significance $(P<0.05)$. (H) Representative images of the decreased cell migration observed between NOS3 and NOS3TR cells. 
reduction of ZEB1 expression in shZEB1 cells was observed by immunoblot. Consistent with the reduction of ZEB1 expression, shZEB1 showed an epithelial cell-like morphology with tight cell-cell adhesion (Figure 6A). After either shCtrl or shZEB1 cells had been injected into the murine peritoneum, the dissemination of cancer cells on the mesothelium was examined (Figure 6B). As shown in Figure $6 \mathrm{C}$, the proliferation of shZEB1 cells in peritoneum was suppressed compared with shCtrl cells. We confirmed that the immunoexpression of ZEB1 was lower in shZEB1cells-formed tumors than those of shCtrl cells. In addition, metastasis to other organs, such as the pancreas, was significantly suppressed by ZEB1 knockdown (Table 1). In accordance with the reduced proliferation and metastasis, mice injected with shZEB1 cells survived for longer than shCtrl-injected mice (Figure 6D).

We finally investigated whether shZEB1 cells were more sensitive to PTX in vivo. Mice were intraperitoneally injected with either shCtrl or shZEB1 cells and were non-treated or treated with $20 \mathrm{mg} / \mathrm{kg}$ of PTX once a week for three weeks (Figure 6E). Mice were sacrificed 4 weeks after tumor cell injection and the total weight of disseminated tumors of each mouse was measured. Although the intravenous injection of PTX did not affect the tumor weight of shCtrl cells, tumor weight of shZEB1 cells was significantly reduced by PTX treatment (Figure 6F). The Tunnel assay of tumor tissues from PTXtreated mice showed that a number of shZEB1 cells were apoptotic, whereas only a small fraction of shCtrl cells were positive for apoptosis (Figure 6G). Table 1 shows the frequency of metastasis in each organ in the absence or presence of PTX treatment. Regardless of PTX treatment, recurrences in the diaphragm and pancreas were more frequently observed in the shZEB1-inoculated group than the Ctrl-inoculated group. These results indicate the pivotal role of ZEB1 in the acquisition of resistance to PTX in vivo.

\section{DISCUSSION}

Peritoneal metastasis is the most frequent clinical presentation of EOC, composed of multiple steps: release from the original ovarian neoplasm, attachment to the mesothelium, and subsequent migration/invasion into
A

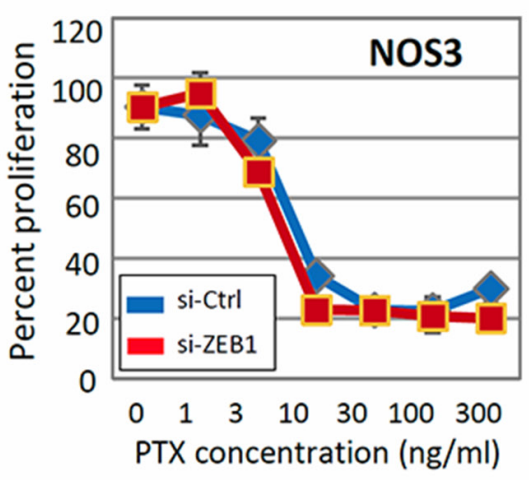

B
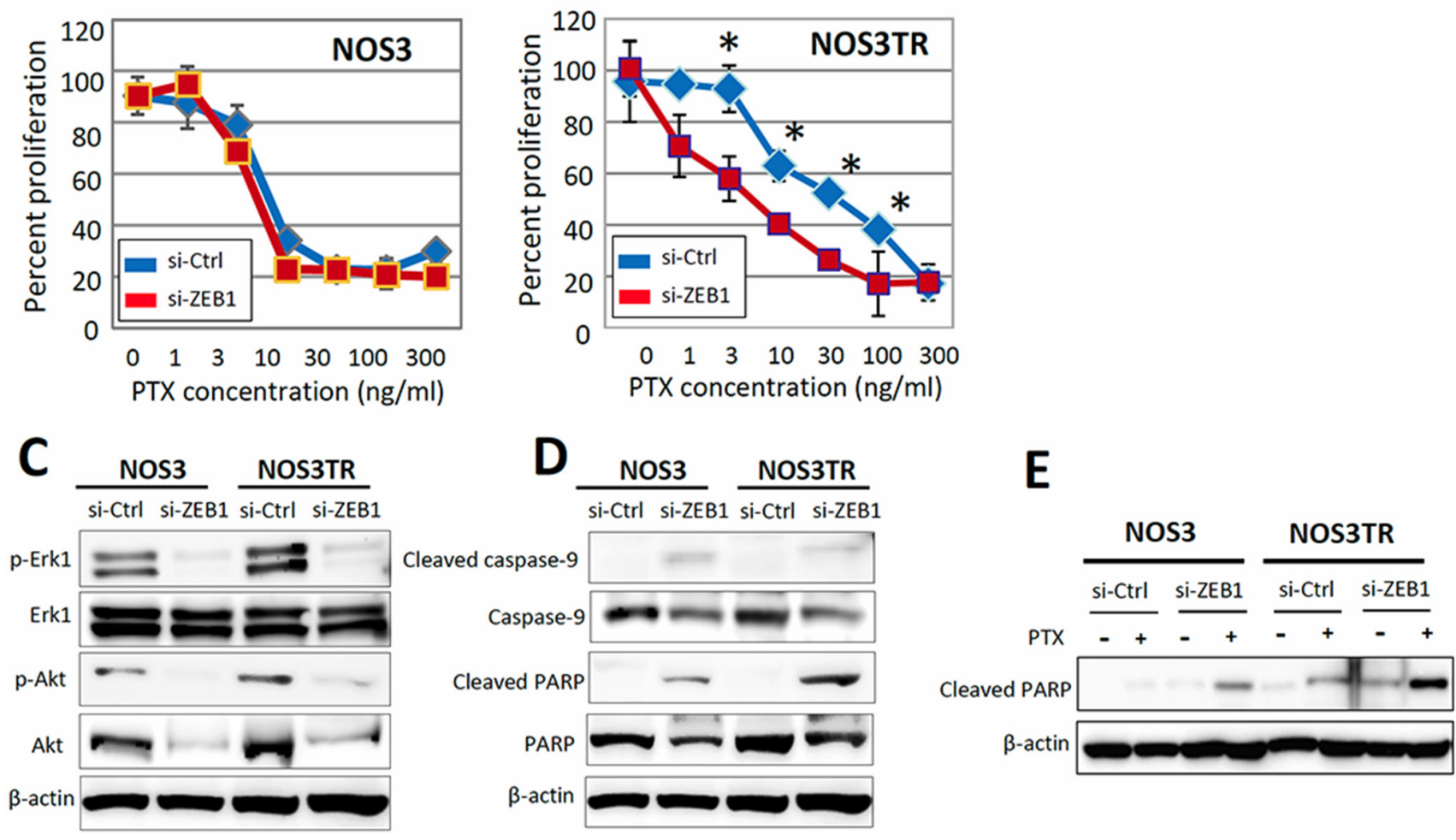

Figure 4: Restoration of PTX-sensitivity by silencing ZEB1 expression in chronic PTX-resistant EOC cells. (A) The PTX-sensitivity curves with or without silencing of ZEB1 in parental NOS3 cells. Asterisks show significance $(P<0.05)$. (B) The PTXsensitivity curves with or without silencing of ZEB1 in parental NOS3TR cells. Depletion of ZEB1 made NOS3TR cells more sensitive to PTX treatment. Asterisks show significance $(P<0.05)$. (C) The expression of phosphorylation of ERK1 and Akt. The phosphorylation of ERK1 and Akt was significantly decreased in ZEB1-depleted NOS3TR cells compared with that of control siRNA-transfected NOS3TR cells. (D) The greater induction of cleaved PARP expression in ZEB1-depleted NOS3TR cells compared with that of NOS3 cells. (E) Significantly higher expression of cleaved PARP in ZEB1-depleted NOS3TR cells in the presence of PTX, compared with that of control siRNA-transfected NOS3TR cells. 
the subperitoneal tissue. Although most EOC patients with peritoneal dissemination are asymptomatic until the late stage, tumor dissemination throughout the peritoneal cavity gradually spreads underneath the surface. On the other hand, as well as peritoneal metastasis, intrinsic or acquired chemoresistance remains a major therapeutic hurdle to improve the prognosis of patients with EOC. PTX, which exerts its effect through the stabilization of microtubules, induction of cell cycle arrest in G2$\mathrm{M}$, and activation of proapoptotic signaling, is a firstline chemotherapeutic agent that is effective for the treatment of EOC $[8,18]$. Although PTX as a front-line antineoplastic agent has contributed to improve the short-term survival, the 5-year survival rate is lower than $30 \%$ in advanced EOC patients [19]. In clinical practice we occasionally encounter sudden recurrences in the peritoneal cavity or lymph nodes during intermittent treatment with antineoplastic drugs, including PTX. The increasing metastatic potential and process of chemoresistance may have concurrently developed during the evolution of tumors toward increasingly malignant characteristics. Considering such molecular mechanisms, we previously revealed that chronic PTX-resistant EOC cells displayed more mesenchymal features, such as the loss of the epithelial phenotype, acquisition of fibroblastlike morphology, switch of EMT-related markers, and enhanced migratory potentials both in vitro and in vivo [8]. However, detailed molecular mechanisms underlying such chemoresistance and subsequent metastasis-promoting potential in EOC have yet to be clarified. In our current analysis, we focused on ZEB1, which is one of the major transcription factors that facilitate EMT and its expression was reported to be associated with a poor clinical outcome in patients with several solid malignancies [9-11].

We first demonstrated that various expression levels of ZEB1 were identified in $82.5 \%$ of the EOC tissue samples. Regarding the correlation between the ZEB1 expression and prognosis, positive ZEB1 expression was a significant predictor of poorer overall survival compared with negative expression. Furthermore, as the positivity of ZEB1 expression incresed, patients accordingly showed poorer overall survival. Earlier studies revealed that the positive expression of ZEB1 in endometrial cancers, colorectal carcinomas, and hepatocellular and
A

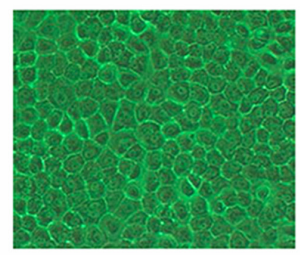

B

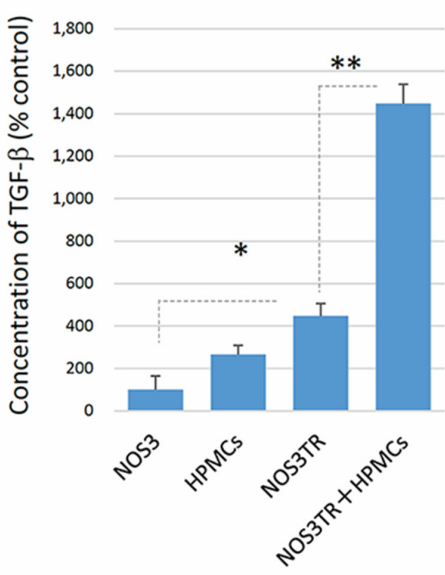

C

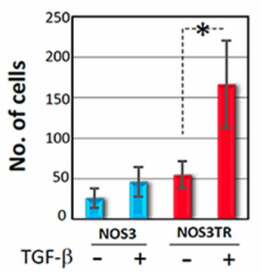

D
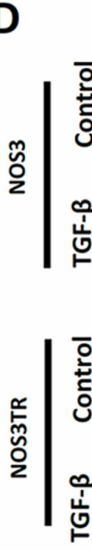
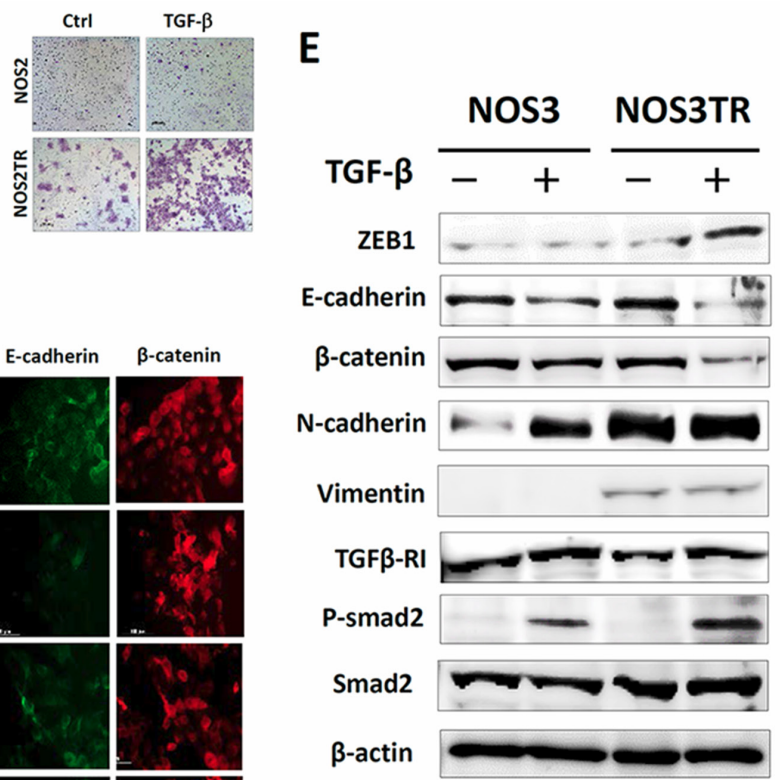

Figure 5: Enhanced ZEB1 expression by microenvironmental production of TGF- $\beta$. (A) The appearance of the cultured human peritoneal mesothelial cells (HPMCs). HPMCs typically showed an epithelial morphology with a cobblestone appearance. (B) TGF- $\beta$ concentrations in conditioned media of HPMCs, NOS3, NOS3TR, and HPMCs plus NOS3TR cells (co-culture). TGF- $\beta$ production in NOS3TR cells was significantly higher than in parental NOS3 cells. Asterisks show significance $(P<0.05)$. (C) The effect of TGF- $\beta$ on migratory potential of parental or PTX-resistant cells. The addition of TGF- $\beta$ to NOS3TR cells led to a significantly enhanced migratory potential compared with parental NOS3 cells (Right panel). Asterisks show significance $(P<0.05)$. Left panel: representative images. (D) The immunofluorescence expressions of E-cadherin and $\beta$-catenin in NOS 3 or NOS3TR cells with or without TGF- $\beta$. The presence of TGF- $\beta$ caused a more mesenchymal cell shape with the loss of cell polarity and decreased E-cadherin expression, particularly in NOS3TR cells. (E) Western blot analysis of the EMT, or TGF- $\beta$ signal-related proteins in NOS3 and NOS3TR cells with or without TGF- $\beta$. The addition of TGF- $\beta$ resulted in increased ZEB1 expression with the enhanced expression of phosphorylated Smad 2, particularly in NOS3TR cells. 
prostate cancer was associated with aggressive disease, poor differentiation, the development of metastases, and a poor clinical prognosis [20-22]. Our current findings are consistent with such prior evidence. Therefore, we could obtain evidence that ZEB1 plays a central role in the progression of EOC. A possible explanation for this finding was that an unfavorable outcome in patients with ZEB1 may be due to the chemoresistance and increased metastatic ability of EOC.

We subsequently examined the molecular mechanism whereby ZEB1 exerted its tumor-promoting effect in EOC in vitro. Using various EOC cell lines, we found that ZEB1 was expressed in highly metastatic EOC cell lines, and the depletion of ZEB1 expression led to downregulation of the migration, invasion, adhesion to mesothelial cells, and PTX sensitivity of EOC cells. Therefore, the results showing that an increase of E-cadherin and decrease of vimentin and $\mathrm{N}$-cadherin were clearly observed by ZEB1 depletion were consistent with the occurrence of mesenchymalepithelial transition. As aforementioned, one of the major therapeutic barriers to improving such a poor clinical outcome is chemoresistance that is related to a multinetwork of molecular mechanisms [23]. In the current examination, the knockdown of ZEB1 significantly restored PTX-sensitivity and reduced motility / invasiveness in chemoresistant cells, suggesting that ZEB1 contributes to the development / maintenance of both the PTX-resistance and metastatic potential of these cells. In other words, ZEB1 may also switch the EOC cell phenotype from non-metastatic to highly metastatic and from chemosensitive to chemoresistant. According to a previous report, ZEB1 was associated with cell sensitivity to conventional chemotherapy, including gemcitabine,
A
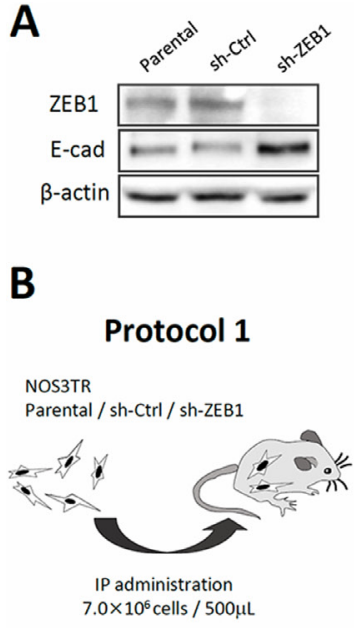

D

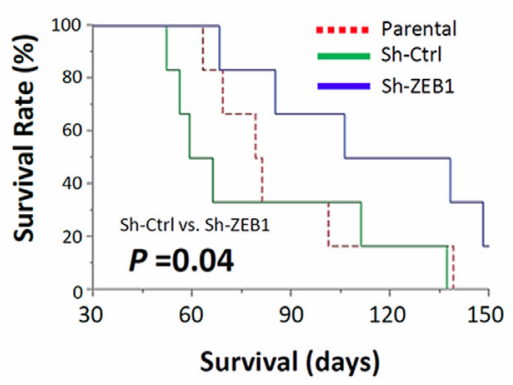

E

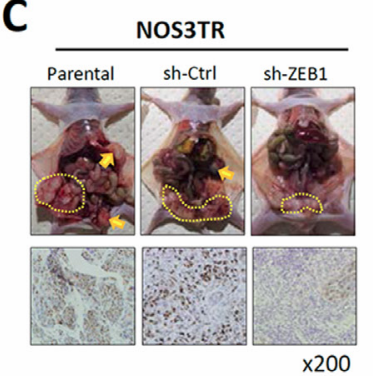

$x 200$

\section{F}

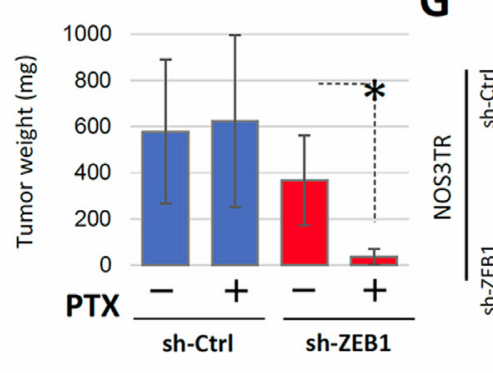

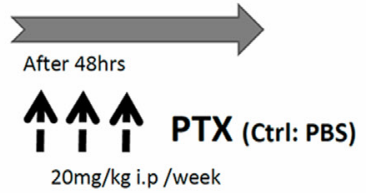

G

PBS PTX

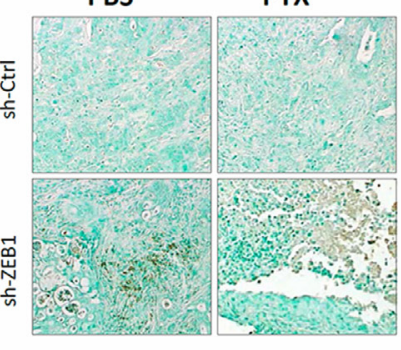

Figure 6: ZEB1 is required for chemo-resistance to PTX in vivo. (A) Generation of NOS3TR cells that constitutively expressed control shRNA (shCtrl) or ZEB1 shRNA (shZEB1) by retrovirus infection (immunoblot). The morphology of shCtrl and shZEB1 cells. shZEB1 showed an epithelial cell-like morphology with clear cell-cell adhesion. (B) The injection of either shCtrl or shZEB1 cells into the murine peritoneum (IP administration: $7.0 \times 10^{6}$ cells / $500 \mu \mathrm{L}$ ). (C) Decreased intraperitoneal dissemination in the shZEB1 cellinoculated mice, compared with the control mice (upper panels). Suppressed expressions of ZEB1 observed in the peritoneal metastatic lesions in the shZEB1 cell-injected mice (lower panels). (D) Survival curves of the parental, shCtrl or shZEB1 cell- inoculated mice. The mice injected with shZEB1 cells survived for longer than the shCtrl-injected mice $(P=0.04)$. (E) The in vivo PTX-sensitivity model. Mice were intraperitoneally injected with either shCtrl or shZEB1 cells, and were treated with or without $20 \mathrm{mg} / \mathrm{kg}$ of PTX once a week for three weeks. Mice were sacrificed 4 weeks after tumor cell injection and the total volume of disseminated tumors of each mouse was measured. (F) Total weight of intraperitoneal disseminated tumors in shCtrl and shZEB1 cell-inoculated mice in the presence or absence of PTX treatment. Asterisks show significance $(P<0.05)$. (G) TUNEL assay of tumor tissues from PTX-treated mice. A number of shZEB1 cells were apoptotic, whereas only a small fraction of shCtrl cells were positive for apoptosis. 
Table 1: The frequency of metastasis in each organ in the absence or presence of the PTX treatment

\begin{tabular}{|c|c|c|c|c|c|c|}
\hline \multirow{2}{*}{ Metastasis } & \multicolumn{7}{c}{ NOS3TR sh-Ctrl } \\
\cline { 2 - 7 } \multicolumn{1}{c|}{} & PBS & PTX & (Total) & PBS & PTX & (Total) \\
\hline Diaphragm & $3 / 4^{*}$ & $2 / 4$ & $(5 / 8)$ & $0 / 4^{*}$ & $0 / 4$ & $(0 / 8)$ \\
\hline Liver & $3 / 4$ & $2 / 4$ & $(5 / 8)$ & $2 / 4$ & $1 / 4$ & $(3 / 8)$ \\
\hline Pancreas & $3 / 4^{* *}$ & $4 / 4^{* * *}$ & $(7 / 8)$ & $0 / 4^{* *}$ & $0 / 4^{* * *}$ & $(0 / 8)$ \\
\hline Spleen & $0 / 4$ & $1 / 4$ & $(1 / 8)$ & $0 / 4$ & $0 / 4$ & $(0 / 8)$ \\
\hline
\end{tabular}

5-fluorouracil (5-FU), and cisplatin in cancer cells [24]. In addition, silencing ZEB1 expression could significantly restore the chemosensitivity of docetaxel-resistant human lung adenocarcinoma cells as well as inhibit their migratory ability through reversing the mesenchymal phenotype [13]. Our current findings are consistent with such earlier evidence. Accordingly, we could reveal that ZEB1 plays a pivotal role in the acquired chemoresistance of EOC cells.

The TGF- $\beta$ signaling pathway is one of the pivotal regulators of the multistep tumor microenvironment related to EMT [25-27]. Similarly to other EMT-inducible markers, such as Snail, Twist, and Slug, ZEB1 was significantly enhanced by the stimulation of TGF- $\beta$ [28]. The interaction between the peritoneal mesothelium and tumor cells plays a major role in ovarian cancer progression. Our data showed that TGF- $\beta$ was produced by EOC cells and it was synergistically upregulated under co-culture conditions with human normal mesothelial cells. Actually, it is plausible that TGF- $\beta$, which is derived from tumor cells, is accumulated in the peritoneal cavity via malignant ascites, and influences mesothelial cells. In our earlier study, an increase of exogenous TGF- $\beta$ induced the additional production of endogenous TGF- $\beta$ from mesothelial cells, resulting in the synergistic accumulation of TGF- $\beta$ [29]. Interestingly, in the current study, TGF- $\beta$ production in the chronic PTX-resistant cells was significantly higher than in naive EOC cells. In addition, the TGF- $\beta$ stimulation led to the synergistic increase of ZEB1 expression with the enhanced expression of phosphorylated Smad 2, particularly in the PTX-resistant cells. These results suggest that the microenvironmental cell-to-cell communication between EOC and the mesothelium contributes to the auto-stimulatory TGF- $\beta$ accumulation and corresponding increase of ZEB1, resulting in the further acquisition of the metastatic potential and/or chronic PTX-resistance of EOC. However, our current data were too preliminary and hypothesisgenerating, to understand the whole picture of TGF- $\beta$ / ZEB1 axis in EOC behavior in the peritoneal cavity. We would like to clarify it through further examinations in a future study.

In summary, these findings suggest that ZEB1 functions as both a chemoresistance- and metastasis- promoting factor in EOC. The overexpression of ZEB1 was found in EOC tissues and chemoresistant EOC cell lines. This is the first report showing that ZEB1 knockdown can enhance the sensitivity of EOC cells to PTX as well as the metastatic potential related to EMT. Furthermore, our data suggest a possible association between the PTX-resistance and enhanced metastatic potential of EOC through the ZEB1-TGF- $\beta$ axis. In our animal model, in accordance with the reduced proliferation and metastasis, mice injected with ZEB1 silencing PTXresistant EOC cells survived for longer than the control cell-injected mice. Although the current findings must be confirmed by other future studies, ZEB1 may be a helpful biological marker and possible target for EOC therapy. Thus, ZEB1 should be explored as a candidate therapeutic target for modulating the paclitaxel sensitivity of EOC. However, one of the most important prognostic factors for EOC is thought to be the resistance to cisplatin. To our knowledge, the association of ZEB1 expression with the cisplatin-resistance has not been clarified in the literature. Unfortunately, we cannot examine this topic in the current study. We would like to elucidate it in the future. At least, the current findings indicate that the immunoreactive identification of ZEB1 expression might be a crucial predictor of patients who will show a poor oncologic outcome, and its identification may lead to the selection of better treatment strategies.

\section{MATERIALS AND METHODS}

\section{Cell culture}

The ES-2, TOV21G, A2780, HEY, SKOV3, OVCAR3, CAOV3, and OV90 cell lines were maintained in RPMI1640 medium with 10\% FBS and penicillin/ streptomycin. These cell lines were obtained from the American Type Culture Collection (Manassas, VA, USA) in 2012-2013. The NOS2 and NOS3 cells, derived from serous EOC, were established in our institute [30, 31]. These cell lines were maintained in RPMI-1640 (Sigma, St. Louis, MO, USA) supplemented with $10 \%$ fetal bovine serum (FBS) and penicillin-streptomycin at $37^{\circ} \mathrm{C}$ in a humidified atmosphere of $5 \% \mathrm{CO}_{2}$. The NOS2TR and NOS3TR cells, established from parental NOS2 and NOS3 
cells, respectively acquired chronic resistance to paclitaxel [32-34]. Human peritoneal mesothelial cells (HPMCs) were isolated from surgical specimens of the human omentum after obtaining consent from each patient and approval of the Ethics Committee of Nagoya University. The procedure for HPMC isolation was described in detail in our previous report [35]. HPMCs were cultured in medium composed of RPMI-1640 (Sigma, St. Louis, MO, USA) supplemented with $10 \%$ FBS and penicillinstreptomycin.

\section{Immunohistochemical staining}

Tissue samples of EOC were obtained after receiving informed consent from patients who were surgically treated at Nagoya University Hospital. Formalin-fixed, paraffin-embedded tissue sections were cut at a thickness of $4 \mu \mathrm{m}$. For heat-induced epitope retrieval, deparaffinized sections in $0.01 \mathrm{M}$ citrate buffer (Target Retrieval Solution, pH 6.1, Dako) were heated three times at $90^{\circ} \mathrm{C}$ for $5 \mathrm{~min}$ using a microwave oven. Immunohistochemical staining was performed using the avidin-biotin immunoperoxidase technique with the Histofine SAB-PO kit (Nichirei, Tokyo, Japan) according to the manufacturer's protocol, and the detailed experimental procedure was described previously [36]. Sections were incubated at $4^{\circ} \mathrm{C}$ for $12 \mathrm{hrs}$ with primary antibody (anti-rabbit- ZEB1 polyclonal, at a 1: 1,000 dilution, Cell Signaling, MA, USA). As a negative control, the primary antibody was replaced with normal rabbit IgG at an appropriate dilution. The intensity of immunostaining for ZEB1 was scored semiquantitatively on a four-tiered scale based on the percent positivity / area of stained cells as follows: For the evaluation of ZEB1 expression, the staining intensity was scored as 0 (negative), 1 (weak), 2 (medium), or 3 (strong). The extent of staining was scored as $0(0 \%), 1(1 \%-10 \%), 2(10 \%-50 \%)$, or $3(51 \%<)$ according to the percentage of the positive staining area relative to the total tumor area. The sum of the intensity and extent scores was used as the final staining score (0-6) for ZEB1. Tumors with a final staining score of 0, 1-2, 3-4, and 5-6 were considered to have negative, weakly, moderately, and strongly positive expression, respectively.

\section{Inhibition of ZEB1 by small interfering RNA (siRNA) and shRNA}

We designed and purchased two different siRNA duplexes of ZEB1, si-ZEB1 (sense, 5'-GCAUCCAAAGAACAAGAAATT-3') and siZEB1-2 (sense, 5'-GCCAACAGUUGGUUUGGUATT, siRNA duplexes (si-Ctrl) (sense, 5'-CAGUCGCGUUUGCGACUGGUU-3'), with the same GC content as ZEB1 siRNA (si-ZEB) from Takara (Tokyo, Japan). The siRNA was used to transfect parental and paclitaxel-resistant EOC cells at a final concentration of $80 \mathrm{nmon} / \mathrm{L}$ using GenePorter-2 (Genlantis, San Diego, CA, USA) according to the manufacturer's protocol.

To generate NOS3TR cells that constitutively expressed the shRNAs, oligonucleotides encoding shRNAs specific for human ZEB1 and luciferase were cloned into the pSIREN-RetroQ vector (Clontech Laboratories, Inc.). The sequences of the shRNAs were: 5'-GCATCCAAAGAACAAGAAA-3' (shZEB1) and 5'-CTTACGCTGAGTACTTCGA-3' (control). Recombinant retrovirus was produced, and infected NOS3TR cells were selected with $1 \mu \mathrm{g} / \mathrm{mL}$ puromycin for 3 days.

\section{PTX chemosensitivity assay}

The PTX chemosensitivity assay was performed as described previously [37]. Briefly, cells were seeded in triplicate in 96-well plates at a density of 5,000 cells in a volume of $200 \mu \mathrm{L}$ of culture media containing $10 \%$ FBS. After incubation for $24 \mathrm{hrs}$ at $37^{\circ} \mathrm{C}$, the medium was replaced with fresh medium with or without various concentrations of PTX (Bristol Myers Squib, Tokyo, Japan). After an additional $72 \mathrm{hrs}$, the cell viability was assayed using the Cell Titer 96 Aqueous One Solution Cell Proliferation Assay kit (Promega Corp., Tokyo, Japan).

\section{In vitro migration and invasion assay}

Cell invasion was evaluated using 24-well Matrigel invasion chambers (Becton Dickinson Labware, Franklin Lakes, NJ, USA). Cells were suspended in the upper chamber at a final concentration of $50 \times 10^{5} / \mathrm{mL}$ in $200 \mu \mathrm{L}$ of RPMI 1640 supplemented with $0.1 \%$ bovine serum albumin (BSA). The lower chamber contained $750 \mu \mathrm{L}$ of RPMI 1640 supplemented with $10 \%$ FBS. After $10 \mathrm{hrs}$ of incubation, the remaining tumor cells on the upper surface of the filters were removed by wiping with cotton swabs, and the invading cells on the lower surface were stained with May-Grünwald Giemsa staining. Cells on the lower surface of the filters were counted under a microscope at a magnification of 200, and we performed four individual experiments using the invasion assay in triplicate.

Cell migration was assayed in 24-well Transwell cell culture chambers (Costar, Corning, NY, USA). Cells were suspended in the upper chamber at a final concentration of $1.0 \times 10^{5} / \mathrm{mL}$ in $200 \mu \mathrm{L}$ of RPMI 1640 medium. The subsequent procedures were the same as those used for the 'Invasion Assay'. In addition, we examined the effect of siRNA transfection on the migration of parental and PTXresistant EOC cells. Cells transfected with siRNAs were seeded onto the upper chamber and allowed to migrate to the fibronectin-coated lower surface for $24 \mathrm{hrs}$. The number of cells that had migrated to the lower surface was counted to evaluate the migratory ability. Cells were seeded in 10-cm dishes in RPMI1640 containing 10\% FBS. After reaching 50\% confluency, the medium was replaced by fresh RPMI 1640 containing 10\% FCS, and 
transfection of siRNA (si-Ctrl and si-ZEB) was performed using GenePorter-2 (San Diego, CA, USA). Sixty hours after transfection, the cells were trypsinized and pelleted. Subsequently, the cells were re-plated in the upper chambers of Transwell plates at a density of $50 \times 10^{4} / \mathrm{mL}$ in $200 \mu \mathrm{L}$ of RPMI 1640. The lower chamber contained $700 \mu \mathrm{L}$ of RPMI 1640 supplemented with $10 \%$ FBS. The subsequent procedure was the same as described above. We performed four individual experiments in which this assay was performed in triplicate.

\section{Adhesion assay of monolayered HPMCs}

HPMCs were trypsinized from $75 \mathrm{~cm}^{2}$ flasks and plated on clear-bottom 96-well microtiter Plates (Falcon, Corning, NY, USA) at 5,000 cells/well. After confluency, the monolayered HPMCs were washed twice with $200 \mu \mathrm{L}$ of serum-free RPMI 1640 medium. Then, cells were plated at a density of $1.0 \times 10^{4}$ cells $/ 200 \mu \mathrm{L}$ volume of serumfree RPMI 1640 medium in each well. Culture plates were centrifuged at $500 \mathrm{rpm}$ for $30 \mathrm{sec}$, and incubated at $37^{\circ} \mathrm{C}$ for $2 \mathrm{hrs}$ in a humidified atmosphere of $5 \% \mathrm{CO}_{2}$. After incubation, the plates were washed three times with the assay buffer to remove non-adherent cells. Then, adherent cells were evaluated for cell-viability using a modified tetrazolium salt MTT assay with the Cell Titer 96 Aqueous One Solution Cell Proliferation Assay kit (Promega Corp., Tokyo, Japan). The adhesion index was calculated using the following formula:

Adhesion index $=$ Absorbance $(492 \mathrm{~nm})$ of transfected cells plated well) - Absorbance (492 nm) of HPMCs alone\}. condition.

The assay was replicated six times for each

\section{Western blot analysis}

The Western blotting procedure was described previously [38]. As primary antibodies, we used anti-Ecadherin (Santa cruz, CA, USA), anti-N-cadherin (Santa Cruz, CA, USA), anti- $\beta$-catenin (Santa Cruz, CA, USA), anti-Fibronectin (Santa Cruz, CA, USA), anti-vimentin (Santa Cruz, CA, USA), anti-ZEB1 (Cell Signaling, MA, USA), anti-ERK1 (Santa Cruz, CA, USA), anti-p-ERK1 (Santa Cruz, CA, USA), anti-Akt (Cell Signaling, MA, USA), anti-p-Akt (Cell Signaling, MA, USA), anti- PARP (Cell signaling, MA, USA), anti-cleaved PARP (Cell Signaling, MA, USA), anti-Caspase 9 (Cell Signaling, MA, USA), anti-cleaved Caspase 9 (Cell Signaling, MA, USA), anti-TGF- $\beta$ R-I (Santa Cruz, CA, USA), anti-pSmad2 (Cell Signaling, MA, USA), anti-Smad 2 (Cell Signaling, MA, USA), anti-Smad4 (Cell Signaling, MA, USA), and anti- $\beta$-actin antibodies (Sigma-Aldrich, MO, USA). The primary antibodies were washed in $0.05 \%$ Tween-20/PBS and then incubated with horseradish peroxidase-conjugated secondary antibody. Proteins were visualized using enhanced chemiluminescence reagent
(Amersham Pharmacia Biotech, NJ, USA). Bands were visualized by ImageQuant LAS 4000 (GE Company, Fairfield, CT, USA).

\section{Immunofluorescence experiment}

Cells were grown in chamber slides (Nalge Nunc International, Rochester, NY, USA). They were fixed for $15 \mathrm{~min}$ with $4 \%$ paraformaldehyde and washed several times with PBS. Coverslips were incubated in blocking solution containing 2\% BSA in PBS for $1 \mathrm{hr}$, and incubated with the appropriate primary antibodies \{anti-mouse E-cadherin and anti-rabbit $\beta$-catenin antibodies (Santa Cruz Biotech, CA, USA) $\}$ for $1 \mathrm{hr}$ at room temperature. After incubations with appropriate secondary antibodies (E-cadherin, goat anti-mouse IgG-FITC; $\beta$-catenin, goat anti-rabbit rhodamine-conjugated IgG: Santa Cruz Biotech, CA, USA), fluorescence was visualized by epifluorescence con-focal microscopy (Pascal, BIORAD, (Hercules, CA, USA).

\section{Treatment with TGF- $\beta$}

NOS2, NOS3, NOS2TR, and NOS3TR cells were stimulated with recombinant human transforming growth factor- $\beta 1$ (TGF- $\beta 1$ ) (R\&D Systems, Minneapolis, MN, USA) at $10 \mathrm{ng} / \mathrm{mL}$ in RPMI-1640 supplemented with $2 \%$ FBS for 96 hrs.

\section{In vivo studies}

Female nude mice (BALB/c) at 6 weeks of age were obtained from Japan SLC (Nagoya, Japan). The treatment protocol followed the guidelines for animal experimentation adopted by Nagoya University. NOS3TR parental, sh-Ctrl-transfected, and sh-ZEB-transfected cells $\left(7 \times 10^{7}\right.$ cells $/ 0.5 \mathrm{~mL}$ of medium/mouse) were injected i.p. to induce the peritoneal metastasis of EOC in the mouse model. The survival time was examined with or without treatment with PTX. In the PTX administration protocol, the intraperitoneal (i.p.) administration of PTX $(20 \mathrm{mg} / \mathrm{kg}$ body weight), was initiated $48 \mathrm{hrs}$ after tumor inoculation, and repeated once a week a maximum of 3 times. Control mice was administered of PBS only. Survival times were compared among these two groups, respectively.

\section{Tunnel assay}

Apoptotic cells were identified using the In Situ Cell Death Detection kit, Fluorescein (Roche Applied Science, Mannheim, Germany), according to the manufacturer's instructions.

\section{Statistical analysis}

All data are expressed as the mean $\pm \mathrm{SD}$. Data were calculated from at least three independent experiments. 
The significance of differences was analyzed by Student's $t$-test. A value of $P<0.05$ was considered to be significant.

\section{Abbreviations}

EMT (epithelial-mesenchymal transition), EOC (epithelial ovarian carcinoma), paclitaxel (PTX).

\section{CONFLICTS OF INTEREST}

All authors declare that there are no conflicts of interest.

\section{REFERENCES}

1. Brun JL, Feyler A, Chene G, Saurel J, Brun G, Hocke C. Long-term results and prognostic factors in patients with epithelial ovarian cancer. Gynecol Oncol. 2000; 78:21-7.

2. Torre LA, Bray F, Siegel RL, Ferlay J, Lortet-Tieulent J, Jemal A. Global cancer statistics, 2012. CA Cancer J Clin. 2015; 65:87-108.

3. Thiery JP. Epithelial-mesenchymal transitions in tumour progression. Nat Rev Cancer. 2002; 2:442-54.

4. Boyer B, Valles AM, Edme N. Induction and regulation of epithelial-mesenchymal transitions. Biochem Pharmacol. 2000; 60:1091-9.

5. Vernon AE, LaBonne C. Tumor metastasis: a new twist on epithelial-mesenchymal transitions. Curr Biol. 2004; 14:R719-21.

6. Kikkawa F, Matsuzawa K, Arii Y, Kawai M, Kobayashi I, Nakashima N, Mizutani S. Randomized trial of cisplatin and carboplatin versus cisplatin, vinblastine and bleomycin in ovarian cancer. Gynecol Obstet Invest. 2000; 50:269-74.

7. Eisenkop SM, Friedman RL, Wang HJ. Complete cytoreductive surgery is feasible and maximizes survival in patients with advanced epithelial ovarian cancer: a prospective study. Gynecol Oncol. 1998; 69:103-8.

8. Kajiyama H, Shibata K, Terauchi M, Yamashita M, Ino K, Nawa A, Kikkawa F. Chemoresistance to paclitaxel induces epithelial-mesenchymal transition and enhances metastatic potential for epithelial ovarian carcinoma cells. Int J Oncol. 2007; 31:277-83.

9. Yang Y, Ahn YH, Chen Y, Tan X, Guo L, Gibbons DL, Ungewiss C, Peng DH, Liu X, Lin SH, Thilaganathan N, Wistuba II, Rodriguez-Canales J, et al. ZEB1 sensitizes lung adenocarcinoma to metastasis suppression by PI3K antagonism. J Clin Invest. 2014; 124:2696-708.

10. Schmalhofer O, Brabletz S, Brabletz T. E-cadherin, betacatenin, and ZEB1 in malignant progression of cancer. Cancer Metastasis Rev. 2009; 28:151-66.

11. Hashiguchi M, Ueno S, Sakoda M, Iino S, Hiwatashi K, Minami K, Ando K, Mataki Y, Maemura K, Shinchi H, Ishigami S, Natsugoe S. Clinical implication of ZEB-1 and E-cadherin expression in hepatocellular carcinoma (HCC). BMC Cancer. 2013; 13:572.
12. Mock K, Preca BT, Brummer T, Brabletz S, Stemmler MP, Brabletz T. The EMT-activator ZEB1 induces bone metastasis associated genes including BMP-inhibitors. Oncotarget. 2015; 6:14399-412. https://doi.org/10.18632/ oncotarget.3882.

13. Ren J, Chen Y, Song H, Chen L, Wang R. Inhibition of ZEB1 reverses EMT and chemoresistance in docetaxelresistant human lung adenocarcinoma cell line. J Cell Biochem. 2013; 114:1395-403.

14. Meidhof S, Brabletz S, Lehmann W, Preca BT, Mock K, Ruh M, Schuler J, Berthold M, Weber A, Burk U, Lubbert M, Puhr M, Culig Z, et al. ZEB1-associated drug resistance in cancer cells is reversed by the class I HDAC inhibitor mocetinostat. EMBO Mol Med. 2015; 7:831-47.

15. Kikuchi M, Yamashita K, Waraya M, Minatani N, Ushiku H, Kojo K, Ema A, Kosaka Y, Katoh H, Sengoku N, Enomoto T, Tanino H, Sawanobori M, et al. Epigenetic regulation of ZEB1-RAB25/ESRP1 axis plays a critical role in phenylbutyrate treatment-resistant breast cancer. Oncotarget. 2016; 7:1741-53. https://doi.org/10.18632/oncotarget.6480.

16. Sanchez-Tillo E, Fanlo L, Siles L, Montes-Moreno S, Moros A, Chiva-Blanch G, Estruch R, Martinez A, Colomer D, Gyorffy B, Roue G, Postigo A. The EMT activator ZEB1 promotes tumor growth and determines differential response to chemotherapy in mantle cell lymphoma. Cell Death Differ. 2014; 21:247-57.

17. Kajiyama H, Shibata K, Terauchi M, Ino K, Nawa A, Kikkawa F. Involvement of SDF-1alpha/CXCR4 axis in the enhanced peritoneal metastasis of epithelial ovarian carcinoma. Int J Cancer. 2008; 122:91-9.

18. Horwitz SB. Taxol (paclitaxel): mechanisms of action. Ann Oncol. 1994; 5:S3-6.

19. Agarwal R, Kaye SB. Ovarian cancer: strategies for overcoming resistance to chemotherapy. Nat Rev Cancer. 2003; 3:502-16.

20. Spoelstra NS, Manning NG, Higashi Y, Darling D, Singh M, Shroyer KR, Broaddus RR, Horwitz KB, Richer JK. The transcription factor ZEB1 is aberrantly expressed in aggressive uterine cancers. Cancer Res. 2006; 66:3893-902.

21. Spaderna S, Schmalhofer O, Wahlbuhl M, Dimmler A, Bauer K, Sultan A, Hlubek F, Jung A, Strand D, Eger A, Kirchner T, Behrens J, Brabletz T. The transcriptional repressor ZEB1 promotes metastasis and loss of cell polarity in cancer. Cancer Res. 2008; 68:537-44.

22. Singh M, Spoelstra NS, Jean A, Howe E, Torkko KC, Clark HR, Darling DS, Shroyer KR, Horwitz KB, Broaddus RR, Richer JK. ZEB1 expression in type I vs type II endometrial cancers: a marker of aggressive disease. Mod Pathol. 2008; 21:912-23.

23. Meads MB, Gatenby RA, Dalton WS. Environmentmediated drug resistance: a major contributor to minimal residual disease. Nat Rev Cancer. 2009; 9:665-74.

24. Arumugam T, Ramachandran V, Fournier KF, Wang H, Marquis L, Abbruzzese JL, Gallick GE, Logsdon CD, 
McConkey DJ, Choi W. Epithelial to mesenchymal transition contributes to drug resistance in pancreatic cancer. Cancer Res. 2009; 69:5820-8.

25. Crosas-Molist E, Bertran E, Fabregat I. Cross-Talk Between TGF-beta and NADPH Oxidases During Liver Fibrosis and Hepatocarcinogenesis. Curr Pharm Des. 2015; 21:5964-76.

26. Dhanasekaran R, Nakamura I, Hu C, Chen G, Oseini AM, Seven ES, Miamen AG, Moser CD, Zhou W, van Kuppevelt TH, van Deursen JM, Mounajjed T, FernandezZapico ME, et al. Activation of the transforming growth factor-beta/SMAD transcriptional pathway underlies a novel tumor-promoting role of sulfatase 1 in hepatocellular carcinoma. Hepatology. 2015; 61:1269-83.

27. Liu J, Chen S, Wang W, Ning BF, Chen F, Shen W, Ding J, Chen W, Xie WF, Zhang X. Cancer-associated fibroblasts promote hepatocellular carcinoma metastasis through chemokine-activated hedgehog and TGF-beta pathways. Cancer Lett. 2016; 379:49-59.

28. Xu J, Lamouille S, Derynck R. TGF-beta-induced epithelial to mesenchymal transition. Cell Res. 2009; 19:156-72.

29. Kajiyama H, Shibata K, Ino K, Nawa A, Mizutani S, Kikkawa F. Possible involvement of SDF-1alpha/CXCR4DPPIV axis in TGF-beta1-induced enhancement of migratory potential in human peritoneal mesothelial cells. Cell Tissue Res. 2007; 330:221-9.

30. Kajiyama H, Kikkawa F, Suzuki T, Shibata K, Ino K, Mizutani S. Prolonged survival and decreased invasive activity attributable to dipeptidyl peptidase IV overexpression in ovarian carcinoma. Cancer Research. 2002; 62:2753-57.

31. Kajiyama H, Shibata K, Ino K, Mizutani S, Nawa A, Kikkawa F. The expression of dipeptidyl peptidase IV (DPPIV/CD26) is associated with enhanced chemosensitivity to paclitaxel in epithelial ovarian carcinoma cells. Cancer Science. 2010; 101:347-54.
32. Kajiyama H, Shibata K, Terauchi M, Yamashita M, Ino K, Nawa A, Kikkawa F. Chemoresistance to paclitaxel induces epithelial-mesenchymal transition and enhances metastatic potential for epithelial ovarian carcinoma cells. International Journal of Oncology. 2007; 31:277-83.

33. Maeda O, Shibata K, Hosono S, Fujiwara S, Kajiyama H, Ino K, Nawa A, Tamakoshi K, Kikkawa F. Spectrin alpha II and beta II tetramers contribute to platinum anticancer drug resistance in ovarian serous adenocarcinoma. International Journal of Cancer. 2012; 130:113-21.

34. Utsumi F, Kajiyama H, Nakamura K, Tanaka H, Mizuno M, Ishikawa K, Kondo H, Kano H, Hori M, Kikkawa F. Effect of Indirect Nonequilibrium Atmospheric Pressure Plasma on Anti-Proliferative Activity against Chronic ChemoResistant Ovarian Cancer Cells In Vitro and In Vivo. Plos One. 2013; 8.

35. Sugiyama K, Kajiyama H, Shibata K, Yuan H, Kikkawa F, Senga T. Expression of the miR200 family of microRNAs in mesothelial cells suppresses the dissemination of ovarian cancer cells. Mol Cancer Ther. 2014; 13:2081-91.

36. Kajiyama H, Hosono S, Terauchi M, Shibata K, Ino K, Yamamoto E, Nomura S, Nawa A, Kikkawa F. Twist expression predicts poor clinical outcome of patients with clear cell carcinoma of the ovary. Oncology. 2006; 71:394-401.

37. Yamashita M, Kajiyama H, Terauchi M, Shibata K, Ino K, Nawa A, Mizutani S, Kikkawa F. Involvement of aminopeptidase $\mathrm{N}$ in enhanced chemosensitivity to paclitaxel in ovarian carcinoma in vitro and in vivo. Int $\mathrm{J}$ Cancer. 2007; 120:2243-50.

38. Hosono S, Kajiyama H, Terauchi M, Shibata K, Ino K, Nawa A, Kikkawa F. Expression of Twist increases the risk for recurrence and for poor survival in epithelial ovarian carcinoma patients. Br J Cancer. 2007; 96:314-20. 